\title{
Designing Bessel Filters Based on Coupled-Resonator Optical Waveguides for Dispersion-Free Slow Light
}

\author{
Hsi-Chun Liu and Amnon Yariv \\ Department of Electrical Engineering and Department of Applied Physics, California Institute of Technology \\ Pasadena, California 91125 \\ hliu@caltech.edu
}

\begin{abstract}
We have developed a formalism for designing Bessel filters based on coupled-resonator optical waveguides (CROWs). The CROWs feature maximally flat group delay and can be realized on different types of resonators.

OCIS codes: (230.4555) Coupled resonators; (230.7370) Waveguides; (230.3120) Integrated optics devices
\end{abstract}

A coupled-resonator optical waveguide (CROW) consists a chain of weakly coupled resonators in which light propagates by tunneling from one resonator to its next-door neighbors [1]. In a CROW, light propagates at a significantly reduced group velocity, which is determined by the inter-resonator coupling. Another unique property of a CROW is its zero group velocity dispersion (GVD) at the center of the band. However, as the frequency approaches the band edge, the GVD increases and approaches infinity, limiting the useful bandwidth of a CROW.

Consider a finite CROW consisting of $N$ resonators coupled to input and output waveguides (Fig. 1). The transmission spectrum $T(\omega)$ can be derived by coupled-mode theory to be an N-th order all-pole filter. Synthesis of optical filters has been studied extensively, most of which are based on the transfer matrix method (TMM) for specific types of resonators such as ring resonators and grating defect resonators [2,3]. In this paper we develop a formalism for extracting the coupling coefficients which is based on coupled-mode theory. These universal coupling coefficients can then be transformed to CROWs of any type of resonator. The filters of particular interest are Bessel filters whose group delay is maximally flat and therefore possess zero GVD across the transmission bandwidth.

The coupled-mode equations of the $N$ resonators in Fig. 1 can be written as [4]

$\frac{d}{d t}\left[\begin{array}{c}a_{1} \\ a_{2} \\ \cdot \\ \cdot \\ \cdot \\ a_{N}\end{array}\right]=-\left[\begin{array}{cccccc}s-i \delta_{1}+\frac{1}{\tau_{e 1}} & i \kappa_{1} & 0 & 0 & \ldots & 0 \\ i \kappa_{1} & s-i \delta_{2} & i \kappa_{2} & 0 & \ldots & 0 \\ 0 & i \kappa_{2} & s-i \delta_{3} & \cdot & \cdot & \cdot \\ \cdot & \cdot & \cdot & \cdot & \cdot & \cdot \\ \cdot & \cdot & . & \cdot & s-i \delta_{N-1} & i \kappa_{N-1} \\ \cdot & \cdot & . & . & i \kappa_{N-1} & s-i \delta_{N}+\frac{1}{\tau_{e 2}}\end{array}\right]\left[\begin{array}{c}a_{1} \\ a_{2} \\ \cdot \\ \cdot \\ \cdot \\ a_{N}\end{array}\right]+\left[\begin{array}{c}\mu_{1} a_{i n} \\ 0 \\ \cdot \\ \cdot \\ \cdot \\ 0\end{array}\right] \equiv-\mu_{1} \boldsymbol{a}_{i n},(1)$

where $s=i\left(\omega-\omega_{0}\right)$ is the detuning of the input frequency from center frequency $\omega_{0}$ and $\delta_{n}=\omega_{n}-\omega_{0}$ is the detuning of individual resonant frequency. $\kappa_{n}$ are the coupling coefficients, $1 / \tau_{e 1}$ and $1 / \tau_{e 2}$ are the external losses of the end resonators to the waveguides, and $\mu_{1}=\sqrt{2 / \tau_{e 1}}$. The intrinsic losses of the resonators are neglected and can be accounted by using predistortion techniques if the loss coefficients are known [5]. The transmission and reflection of the CROW at steady state are respectively

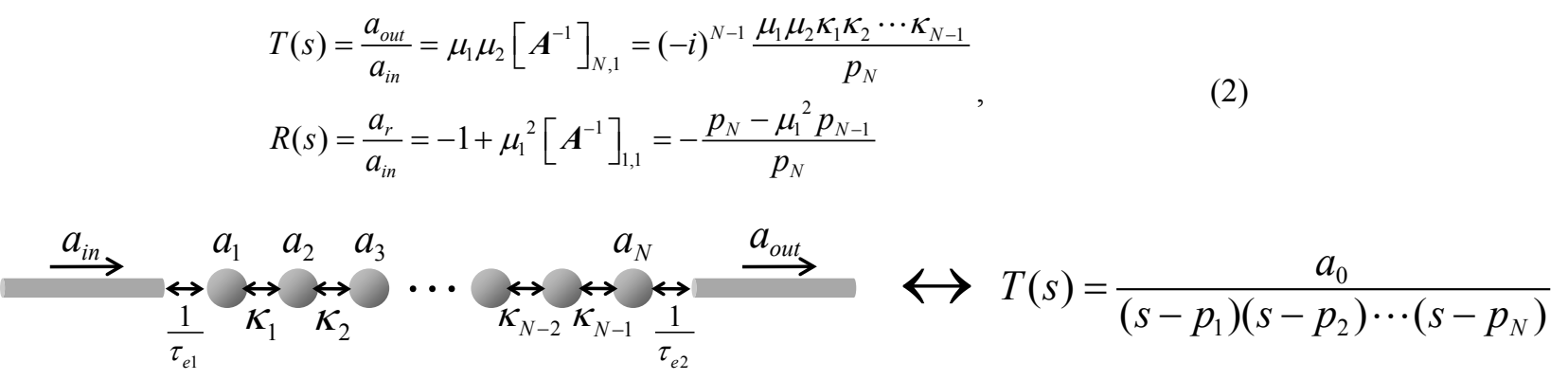

Fig. 1. Transformation between an N-resonator CROW and an N-th order all-pole filter. 


\section{CWM6.pdf}

where $p_{n}$ denotes the determinant of the $n \times n$ bottom-right submatrix of $\boldsymbol{A}$ and is an n-th order polynomial with a leading coefficient of 1 . Therefore, the transmission $T(s)$ is an N-th order all-pole filter. The polynomials $p_{n}$ obey the recursive formulas listed in Table 1. When both $p_{N}$ and $p_{N-1}$ are known, all the frequency detuning $\delta_{n}$ and coupling coefficients $\kappa_{n}$ can be extracted by the recursive formulas. For a desired $T(s)$, the reflection $R(s)$ is also needed in order to find $p_{N-1} . R(s)$ can be found by using $|T(j \omega)|^{2}+|R(j \omega)|^{2}=1$ for lossless systems and using power spectral factorization. There are $2^{N}$ solutions of $R(s)$, the most interesting of which is the one that corresponds to zero frequency detuning $\delta_{n}$ and nearly symmetric $\kappa_{n}$.

Table 1. (Left) Recursive formulas of $p_{n}$. (Right) Transformation of coupling coefficients.

\begin{tabular}{|ll|l|}
\hline$p_{N}=\left(s-i \delta_{1}+\frac{1}{\tau_{e 1}}\right) p_{N-1}+\kappa_{1}^{2} p_{N-2}$ & $\begin{array}{l}\text { Extracting coupling coefficients: } \\
\text { divide } p_{N} \text { by } p_{N-1}:\end{array}$ & $\begin{array}{l}\text { Transformation of coupling coefficients: } \\
\text { Microring CROWs: } \eta=\sin \left(2 \pi \frac{\kappa}{\omega_{F S R}}\right)\end{array}$ \\
$p_{N-1}=\left(s-i \delta_{2}\right) p_{N-2}+\kappa_{2}^{2} p_{N-3}$ & $\left\{\begin{array}{l}\text { quotient }=s-i \delta_{1}+\frac{1}{\tau_{e 1}} \\
\text { remainder }=\kappa_{1}^{2} p_{N-2}\end{array}\right.$ & Grating CROWs: $L=-\frac{1}{\kappa_{g}} \ln \left(\frac{\kappa}{\omega_{g}}\right)$ \\
$p_{2}=\left(s-i \delta_{N-1}\right) p_{1}+\kappa_{N-1}^{2}$ & $\vdots$ & divide $p_{N-1}$ by $p_{N-2} \Rightarrow p_{N-3}$ \\
$p_{1}=s-i \delta_{N}+\frac{1}{\tau_{e 2}}$ & $\vdots$ & $\kappa_{g}:$ grating strength, $\omega_{g}=\frac{\kappa_{g} c}{n}$ \\
\hline
\end{tabular}

In what follows we consider an $\mathrm{N}=6$ Bessel CROW filter as an example. The extracted coupling coefficients are $\left(1 / \tau_{e 1}, \kappa_{1}, \kappa_{2}, \cdots, \kappa_{N-1}, 1 / \tau_{e 2}\right)=(2.068,1.198,0.393,0.397,0.803,1.486,2.427) \cdot B$, where $B$ is the chosen bandwidth of the filter. Fig. 2 illustrates a microring CROW, where the coupling is controlled by the dimensionless coupling coefficients $\eta$, and a grating CROW, where the lengths of the grating sections $L$ control the coupling. The formulas to transform the coupling coefficients are listed in Table 1 . Choosing $B=10 \mathrm{GHz}$, the coefficients of the microring CROW are $\left(\eta_{\text {in }}, \eta_{1}, \eta_{2}, \cdots, \eta_{\text {out }}\right)=(0.696,0.187,0.0617,0.0623,0.126,0.231,0.736)$, while the coefficients of the grating CROW are $\left(L_{1}, L_{2}, \cdots L_{N+1}\right)=(20.3,46.1,57.257 .1,50.1,43.9,19.5) \mu \mathrm{m}$ for $\kappa_{g}=0.1 / \mu \mathrm{m}$. Shown in Fig. 3, the transmission and delay spectra of the two realizations of CROWs are nearly identical, since they have the same coupling coefficients. The group velocity is $c / 62.5$ for the microring CROW and $c / 76.5$ for grating CROW. The group delay is flat across the $10 \mathrm{GHz}$ bandwidth.

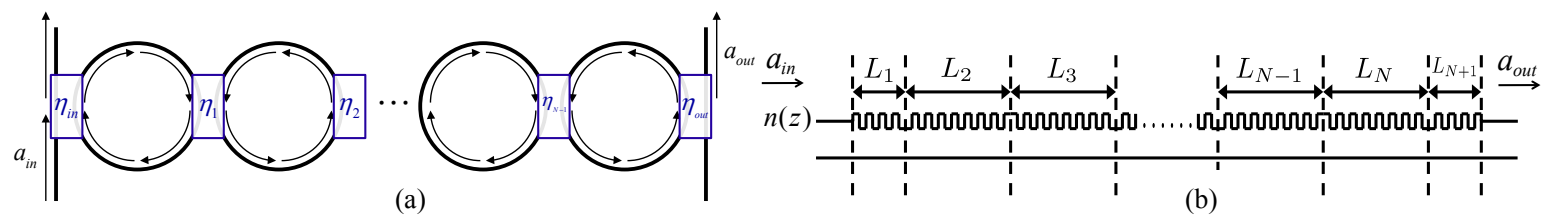

Fig. 2. (a) A microring CROW and (b) a grating defect CROW.
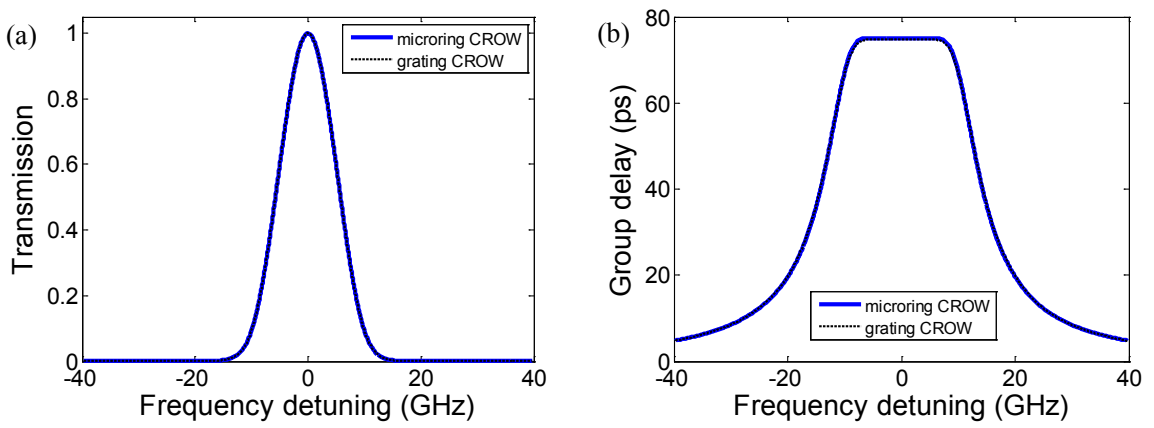

Fig. 3. (a) Transmission spectrum and (b) group delay spectrum of Bessel CROWs.

[1] A. Yariv, Y. Xu, R. K. Lee, and A. Scherer, “Coupled-resonator optical waveguide: a proposal and analysis,” Opt. Lett. 24, 711-713 (1999)

[2] R. Orta, P. Savi, R. Tascone, and D. Trinchero, "Synthesis of multiple-ring-resonator filters for optical systems," IEEE Photon. Technol. Lett. 7, 1447-1449 (1995).

[3] A. Melloni, M. Martinelli, "Synthesis of direct-coupled-resonators bandpass filters for WDM systems," J. Lightwave Technol. 20, 296-301 (2002).

[4] H. A. Haus, Waves and Fields in Optoelectronics (Prentice-Hall, 1984).

[5] A. M. Prabhu and V. Van, "Predistortion techniques for synthesizing coupled microring filters with loss," Opt. Commun. 281, 2760-2767 (2008). 